\title{
The Effect of Topical Application of Lavender Essential Oil on Pain Se- verity during Intravenous Catheterization: A Randomized Clinical Trial
}

\author{
Hamide Jometondoki ${ }^{1}$, Fereshteh Ghorat ${ }^{2}$, Mohammad Hassan Rakhshani ${ }^{3}$, Hasan \\ khalili $^{4 *}$
}

1. Master student medical-sergical in Nursing, Sabzevar University of Medical Science, Sabzevar, Iran.

2. Research Center for Traditional and Alternative, Sabzevar University of medical sciences,Sabzevar, Iran.

3. Department of Biostatistics, School of public health, Sabzevar University of Medical Sciences, Sabzevar, Iran.

4. Department of Nursing, School of Nursing and Midwifery, Sabzevar University of Medical Science, Sabzevar, Iran.

*Correspondence: Department of Nursing, School of Nursing and Midwifery, Sabzevar University of Medical Science, Sabzevar, Iran.

Tel: +98-9151707103. Email: khalili894@gmail.com

Received Sep 01, 2018; Accepted July 20, 2019

\section{Abstract}

Background: Intravenous catheterization is one of the most common invasive interventions in the nursing profession which is associated with pain and patient dissatisfaction. The aim of the present study was to investigate the effect of topical lavender essential oil on the severity of pain caused by intravenous catheterization.

Methods: This randomized clinical trial performed on 66 elective surgery candidates in Shahid Beheshti Hospital of Sabzevar in 2017. The eligible patients was recruited through convenient sampling method considering inclusion criteria they were then randomly allocated into intervention $(n=33)$ and placebo $(n=33)$ groups using permutation blocks. 3 puffs of lavender essential oil were used onto the insertion sites of patients in the intervention group, while in the placebo group there were applied 3 puffs of distilled water. Severity of pain was measured using Visual Analogue Scale (VAS) at immediate, 5 and 10 minutes after intravenous catheterization. Data was presented descriptive statistics and analyzed using Chi-square and Mann-Whitney in SPSS-16. The P-value less than 0.05 considered significant.

Results: The mean score of pain severity in the intervention group immediately, 5 minutes and 10 minutes after the intravenous catheterization was $3.00 \pm 2.23,1.36 \pm 1.08$ and $0.51 \pm 1.14$, while 
in patient of placebo group was $5.72 \pm 2.46,3.12 \pm 2.23$ and $1.8 \pm 1.50$ respectively, there was a statistically significant difference between groups.

Conclusions: The present study showed that the use of topical lavender essential oil is effective in decreasing pain severity caused by the intravenous catheter insertion. Therefore, the topical lavender essential oil can be used to prevent pain in patients before catheter insertion.

Keywords: Lavandula, Catheterization, Central Venous, Pain

$\underline{10.29252 / \mathrm{jgbfnm} \cdot 16.2 .52}$

\section{Introduction}

Pain causes fear, anxiety and disability in people more than any other diseases, moreover it is regarded as the most common cause of health care researches and clinical complaints in patients $(1,2)$. According to the definition of the International Association for the Study of Pain (IASP), pain is an unpleasant sensory and emotional experience associated with actual or potential damage to tissues (3). Different factors may result in pain perception in people. In clinical situations, pain can be caused by diagnostic and therapeutic procedures including skin perforation using a needle to inject (4). More than 500 million cases of intravenous catheterization and more than 25 million instances of intravenous catheterization are annually carried out in American hospitals in the world (5). intravenous catheterization insertion is a common invasive intervention in nursing profession that causes pain, stress and dissatisfaction in patients (6). The initial experience of pain in patients may both create a poor attitude towards treatment and cause fear or avoidance of other therapies (7). The lack of effective pain relief methods can lead to severe disturbances in the relationship between patient and nurse and to decreases patient satisfaction with nursing care (8). Therefore, paying attention to painful clinical procedures and the use of appropriate nursing interventions to relieve pain and anxiety are important tasks of treatment staff (9). Pharmaceutical and non- pharmaceutical methods are now widely used to reduce pain associated with venipuncture including focus deviation, Lidocaine injection and Eutectic Mixture of Local Anesthetics (EMLA) cream. The use of any of the mentioned methods may cause serious complications and limitations (10). The results of a research by Deguzman et al. indicated that intradermal buffer injection of lidocaine $1 \%$ will generate more effective analgesia than normal saline (11). Both methods may cause anxiety and illness as a result of injury and pain (10). In this regard, the results of another study on the topical application of spray onto the catheter insertion site by Hartstein et al. also indicated no significant relationship between the pain severity in the experimental and the control group (12). In practice, the most appropriate method provides the expected analgesia at the shortest time and the lowest cost (13). As for the nursing care in many health and treatment centers, the use of complementary medicine is now widely increasing as an affordable, easy and limited treatment with side effects. According to the definition of the World Health Organization (WHO), the herbal medicine is a crucial component of complementary medicine that has been accepted by a large number of people in the society so that $40 \%$ of commonly used drugs are currently derived from plants and natural resources (14). Lavender with the scientific name, Lavandula angustifolia, is a herbaceous, fragrant, perennial and evergreen plant, belonging to the lamiaceae family which is found in Mediterranean regions of Africa and India (15). The essential oil of this 
plant contains 93 known compounds including Linalool and Linalyl acetate that cause the localized analgesic effect of lavender (16). Jagger believes that Linalyl acetate and Linalool are absorbed 5 minutes after Lavender oil application onto the skin so that they can be detected in the bloodstream with the maximum rate of concentration after 20 minutes (17). The results of another herbal medicine- related research by Asgari et al. indicated that the use of lavender essential oil onto the catheter entrance site could be effective in reducing the pain severity during insertion of arterial needles in patients undergoing hemodialysis (18). Meanwhile, Vakilian et al. conducted a study with the aim to survey the effect of lavender essential oil to properly care for postpartum episiotomy wound and found that the mean score of pain was not significantly different in the Betadine and lavender groups on the fifth day (15). Few studies have investigated the effects of lavender in inhalation form on the pain severity of intravenous catheter insertion. However, there are few studies on analgesic effects of topical lavender on acute pains such as intravenous catheterization. In this regard, the present study aimed to investigate the effect of essential oil of topical lavender on pain severity caused by the intravenous catheter insertion in patients undergoing elective surgery.

\section{Methods}

This Randomized Clinical Trial was done on 66 patients undergoing elective surgery at surgical wards of Emdad Shahid Beheshti Hospital of Sabzevar in 2017. According to study of Asgari et al. (17) and with power $80 \%$ the following formula, 59 participants were calculated. Taking into account the loss to follow up of \%10, a total of 66 samples was estimated.

$$
n=\frac{2 s_{p}^{2}\left(z_{1-\alpha / 2}+z_{1-\beta}\right)^{2}}{d^{2}}=\frac{2 \times 2.80585 \times(1.96+1.28)^{2}}{(1)^{2}} \cong 59
$$

The inclusion criteria were; age between 18-65 years, being completely consciousness and having the ability to establish verbal communication; lack of using analgesic, sedative and antianxiety drugs at least 6-8 hours before venipuncture; not be addicted to any drugs; and not be allergic to lavender essential oil. The exclusion criteria included pain and discomfort before intravenous line insertion; psychological disorders; neuropathy or any other illness that affects pain perception. The data collection tool was demographic information form and Visual Analogue Scale (VAS), VAS is a $10 \mathrm{~cm}$ line of which one end belongs to the absence of pain (Score 0) and the other end shows the most severe pain (Score 10). The patients should encode their pain severity through this scale. VAS is a standard and easy-to-use tool which used in a numerous researches. In a study by Asgari et al. the kappa coefficient of pain severity was 0.948 (4).

After explaining the purpose and method of the research, informed consent was obtained to the participants who were willing to participate in the study. The eligible patients was recruited through convenient sampling method considering inclusion criteria and they were then randomly allocated into intervention $(n=33)$ and placebo $(n=33)$ groups using permutation blocks. After admitting patients into the surgical ward, they transferred into the venipuncture room and lay down on the back in a comfortable position for intravenous catheterization. Lavender essential oil and distilled water were already prepared in similar containers in terms of shape and size. The lavender essential oil, produced by Barij essence Kashan Co, was prepared by distillation and water vapor. The participants, assessor (assistant researcher) and statistician were blind which participants was allocated in the intervention and the placebo groups. The patients in intervention

J Res Dev Nurs Midw, Volume 16, Number 2, December, 2019 
groupreceived 3 puffs $(0.3 \mathrm{ml})$ of Lavender essential oil $(100 \%)$ on the venipuncture site topically 5 minutes before intravenous catheterization (18). In the placebo group, 3 puffs $(0.3 \mathrm{ml})$ of distilled water were used. After 5 minutes, the site was disinfected from center to outside by Betadine; and the catheter was inserted and fixed in the patient's hands. The whole venipuncture procedure was equally performed by the researcher according to the mentioned principles in the reference books in all samples by Malaysian catheter 20 and finally the catheter was fixed with anti-allergenic adhesives. After fixing the catheter the researcher assistant, who was unaware of intervention, asked the patients to mark the pain severity that they had felt during the catheterization, and after 5 and 10 minutes on a VAS. Finally, the collected data was analyzed using SPSS 16 according to the descriptive and inferential tests (t-test, Chi-square test, Fisher's exact test, and Mann-Whitney test). Significance level of all tests was considered as $\mathrm{p}<0.05$.

\section{Results}

The mean age of patients was $38.61 \pm 12.84$ years in the intervention group and $43.67 \pm 14.38$ years in the placebo group. Majority of them was married (90.9\%), housewives (37.9\%), had history of hospitalization $(69.7 \%)$, and lived in the city $(60.6 \%)$.

The results indicated that there was no significant difference between patients in two groups in terms of variables such as gender, marital status, educational attainment, occupational status, hospitalization history and the number of admissions; thus, regarding the mentioned variables, both groups were homogeneous. (Table 1)

Table 1. The Distribution of baseline variables by intervention and placebo groups

\begin{tabular}{|l|c|c|c|}
\hline Group & $\begin{array}{c}\text { Placebo } \\
\text { SD } \pm \text { Mean }\end{array}$ & $\begin{array}{c}\text { Intervention } \\
\text { SD } \pm \text { Mean }\end{array}$ & P value \\
\hline Age & $38.61 \pm 12.84$ & $43.67 \pm 14.38$ & 0.137 \\
\hline BMI & $25.83 \pm 4.71$ & $25.02 \pm 3.55$ & 0.431 \\
\hline
\end{tabular}

The mean scores pain of patients in intervention group immediately, 5 and 10 minutes after intravenous catheterization were $3.00 \pm 2.23,1.36 \pm 1.81$, and $0.51 \pm 1.14$ respectively. The mean pain scores of patients in intervention group immediately, 5 and 10 minutes after intravenous catheterization in the placebo group were 5.72 $\pm 2.46,3.12 \pm 2.23$, and $1.8 \pm 1.50$ respectively in the placebo group. Results of the Mann- Whitney test indicated that there was a significant difference between the severity of pain score among the patients in two groups immediately, 5 and 10 minutes after the intravenous catheterization (Fig. 1). The severity of patients' pain score immediately after the intravenous catheterization $(\mathrm{p}<0.001), 5$ minutes after the venipuncture $(\mathrm{p}<0.001)$ and 10 minutes after the venipuncture $(\mathrm{p}<0.001)$ in the intervention group was significantly less than the placebo group. (Table 2) 
Table 2. The mean of patients' pain severity in three time points after the intravenous catheterization in the two groups

\begin{tabular}{|l|c|c|c|}
\hline \multicolumn{1}{|c|}{ Group } & $\begin{array}{c}\text { Intervention } \\
\text { Mean } \pm \text { SD }\end{array}$ & $\begin{array}{c}\text { Placebo } \\
\text { Mean } \pm \text { SD }\end{array}$ & Test result \\
\hline Immediately after intravenous catheterization & $3.00 \pm 2.23$ & $5.72 \pm 2.46$ & $\mathrm{P}<0.001$ \\
\hline 5 minutes after the intravenous catheterization & $1.36 \pm 1.81$ & $3.12 \pm 2.23$ & $\mathrm{P}<0.001$ \\
\hline 10 minutes after the intravenous catheterization & $0.51 \pm 1.14$ & $1.8 \pm 1.50$ & $\mathrm{P}<0.001$ \\
\hline
\end{tabular}

As shown in Figure 1, the mean score of patients' pain severity was lower in the intervention group than the placebo group immediately, 5 and 10 minutes after the intravenous catheterization.

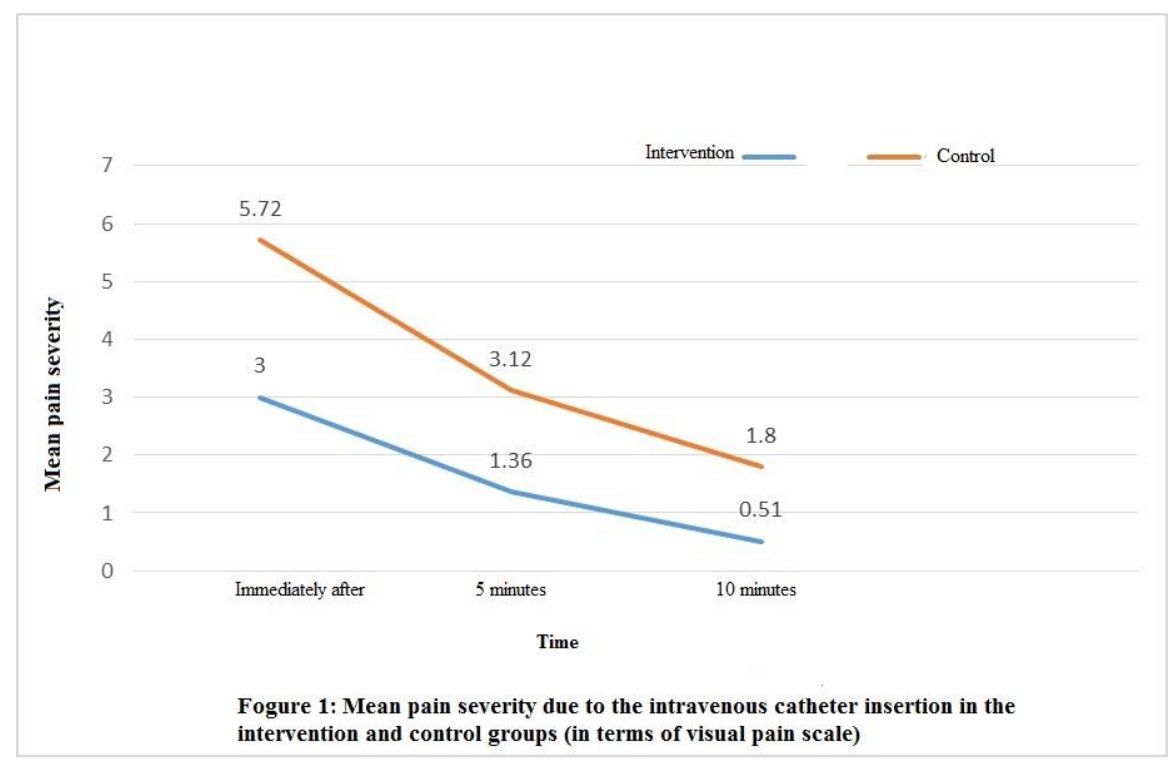

\section{Discussion}

The findings of the present study indicated that applying topical lavender essential oil onto the catheter insertion site indicated a positive effect on the pain severity of intravenous catheterization in patients undergoing elective surgery. The mean score of pain severity of the patients at all three time points (immediately, 5 and 10 minutes after the intravenous catheterization) and was recorded significantly lower in the intervention group (lavender essential oil) than in the placebo group. It is in accordance with the study results of Asgari et al. which indicated that the use of topical lavender essential oil in the arterial needle entry site in 
hemodialysis patients decreased the pain severity of needle entry (18). Beyk Moradi et al. illustrated that the pain severity was lower in children in the intervention group who received Lavender Essence immediately, 5 and 10 minutes after the venipuncture (19). The major findings of their study were also consistent with the present study. Also, Bagheri et al. found that the inhalation aromatherapy with lavender $10 \%$ affected the pain caused by the insertion of arterial needles in patients undergoing hemodialysis (20). Moreover, in another similar study, Tougba Karman et al. demonstrated that inhaling gas fumes with dimensions of $5 \times 5$ along with 2 drops of lavender essential oil 1\% decreased patients' pain and anxiety during venipuncture as well as increasing their satisfaction (21). In another study with the aim of investigating the effect of aromatherapy with lavender essential oil on the level of stress and pain severity caused through the needle insertion by Sion Kimet et al. (2011), the results indicated that inhalation of lavender essence along with oxygen and through a mask could decrease the stress and pain severity during the needle insertion (22); and the results were consistent with the present study. However, the present study was not consistent with results of a research by Vakilian et al. In their study entitled "the effect of lavender essential oil on the Postpartum episiotomy wound care", they discovered that the mean score pain was not statistically different in the Betadine and lavender group on the fifth day (15), probably due to the method of using lavender or the use of low dose medication, or due to the difference in the extent of injury (episiotomy due to the intravenous catheterization).

We aware that a number of limitations may have influenced the results obtained. These limitations included the different threshold of pain in different people; and the participants' cultural; social of psychological factors which affected the pain severity due to the intravenous catheterization, but the researcher could not placebo the above-mentioned factors.

\section{Conclusion}

Overall, knowledge level regarding HPV vaccination among the medical sciences students in southwest of Iran was low. Less than half of the respondents had positive attitude towards HPV vaccination. However, more than half of them were willing to get HPV vaccine.

As future health professionals, medical sciences students play a pivotal role in spreading awareness among a wide range of population. Considering that all participants were studied at medical sciences university and most of them have obtained information about HPV vaccine and cervical screening from medical courses at the university, it is predictable that the awareness level of HPV vaccination will be even lower in the general population. It is necessary to implement sexual health education programs on cervical cancer, HPV infection and HPV vaccination for both the public and the students. Inclusion of HPV vaccine in the National Program on Immunization would also be an effective strategy for improving HPV vaccination and access.

\section{Acknowledgements}

The present paper was derived from a master thesis in the field of medical-surgical nursing which approved by ethical board of the deputy of research at Sabzevar University of Medical Sciences (code: IR.MEDSAB.REC.1396.84) and registered at the Iranian Registry of Clinical Trials (IRCT20171226038078N2). We hereby present the researchers' gratitude to the research 
deputy of Sabzevar University of Medical Sciences, the nurses and all the patients who participated in the research.

\section{References}

1. Mirtajadini H, Kalroozi F, Pishgooie AH. Shiatsu massage and the pain intensity of venipunctury in patients undergoing hemodialysis. MCS. 2016;3(1):27-33. [persian]

2. Vosoughi N, Mitrachehrzad M, Abootalebi GH, Atrkar Z. The effects of distraction on physiological parameters and pain of venipuncture in hospitalized children 3 to 6 years old. Journal of hayat. 2011;16(3,4):39-47. [persian]

3. Sadeghi T, Shamshiri M, Mohammadi N, Shoghi M. Effect of distraction on children's behavioral responses to pain during iv catheter insertion. Journal of hayat. 2013;18(4):19.[persian]

4. Asgari MR, Bakhtiary A, Ebrahimian A, Javadifar K. The effect of different types of transcutaneous electrical nerve stimulations (TENS) on severity of pain related with insertion of intravenous catheter (Angiocut). J Gorgan Univ Med Sci. 2009;10(4):11-17. [persian]

5. Taghinejad H, Asadizaker M, Tabesh H. A comparative analysis of the effects of Betadine and Alcohol as sterilizers on the complications of intravenous catheters. SJIMU. 2005; 13(4):10-17. [persian]

6. Halm MA. Effects of local anesthetics on pain with intravenous catheter insertion. AJCC. 2008; 17(3):265-68.

7. Ghods A.A, Hoseini Abforosh N, Ghorbani R, Asgari M.R. Effect of lavender inhalation on pain intensity during insertion of vascular needles in hemodialysis patients. JBUMS. 2014; 16(10):714. [persian]

8. Bagherian S, Borhani F, Abbaszadeh A, Tehrani H, Pashandi Sh. The severity of pain in children with thalassemia during venipuncture and prior to blood transfusion. Journal of Health \& Development. 2012; 1(2):138-46. [persian]

9. Borhani F, Bagherian S, Abaszadeh A, Ranjbar H, Tehrani H, Soleimanizadeh L. Correlation between anxiety and pain due to intravenous catheters in children with thalassemia. Sci J Iran Blood Transfus Organ. 2012; 9(2):170-4.[persian]

10. Khalili Shomia S, Safavi M, Yahyavi S.H, Farahani H. Assessment of the effect of emla cream on vein puncture pain severity with vein catheter in the patients undergoing cesarean section: randomized placebo controlled trial. J Mazandaran Univ Med Sci. 2011; 22(91):82-8. [persian]

11. Deguzman ZC, O'Mara SK, Sulo S, Haines T, Blackburn L, Corazza J. Bacteriostatic normal saline compared with buffered $1 \%$ lidocaine when injected intradermally as a local anesthetic to reduce pain during intravenous catheterinsertion. J Perianesth Nurs. 2012; 27(6):399-407. 
12. Hartstein B.H, Barry J.D. Mitigation of pain during intravenous catheter placement using a topical skin coolant in the emergency department. Emerg Med J. 2008; 25(5):257-61.

13. Hatmizadeh M, Dalvandi A, Rahgoi A, Ranjbar H. Comparsion of the effect vapocoolant spray and EMLA cream in reduction of anxiety due to intravenous cannulation: A Controlled Randomized Clinical Trial Iranian. JPEN. 2016; 2(4):28-35. [persian]

14. Jahdie F, Haggani H, Kheyrkhah M, Eghdampour F. The effect of calendula ointment in pain intensity of episiotomy among primiparous women. The Journal of Urmia Nursing and Midwifery Faculty. 2011; 9(4):245-52. [persian]

15. Vakilian K, Attarha M, Bekhradi R, Ghebleh F, Hatami Z, ceraj A. The effect of lavender in care of postpartum episiotomy wounds. J Shahrekord Univ Med Sci. 2008; 10(3):63- 9. [persian]

16. Bikmoradi A, Khaleghverdi M, Cheraghi F, Seddighi I, Moradkhani Sh, Soltanian AR. The effect of aromatherapy with lavender essence on physiological indicators of hospitalized preschool children with intravenous catheter insertion: a single-blind clinical trial. Avicenna J Nurs Midwifery Care. 2016; 24(2):76-85. [Persian]

17. Jager W, Buchbauer G, Jirovetz L, Fritzer M. Percutaneous absorption of lavender oil from a massage oil. J Soc Cosmet Chem. 1992; 43(1):49-54.

18. Ghods A.A, Hoseini Abforosh N, Ghorbani R, Asgari M.R. The effect of topical application of lavender essential oil on the intensity of pain caused by the insertion of dialysis needles inhemodialysis patients: A randomizedclinical trial. Complementary Therapies in Medicine. 2015; 23(3):325-30. [persian]

19. Bikmoradi A, Khaleghverdi M, Seddighi I, Moradkhani Sh, Soltanian A, Cheraghi F. Effect of inhalation aromatherapy with lavender essence on pain associated with intravenous catheter insertion in preschool children: A quasi-experimental study. Complementary Therapies in Clinical Practice. 2017; 24(2):85-91.

20. BagheriNesami M, Espahbodi F, Nikkhah A, Shorofi S.A, Yazdani Charati J. The effects of lavender aromatherapy on pain following needle insertion into a fistula in hemodialysis patients. Complementary Therapies in Clinical Practice. 2014; 20(1):1-4.

21. Karaman T, Karaman S, Dogru S, Tapar H, Sahin A, Arici S, Et al. Evaluating the efficacy of lavender aromatherapy on peripheral venous cannulation pain and anxiety: A prospective, randomized study. Complementary Therapies in Clinical Practice. 2016; 23:64-8.

22. Kim S, Kim H.j, Yeo J.S, Hong S.J, Lee J.M, Jeon Y. The effect of Lavender Oil on Stress,Bispectral Index and needle insertion pain in volunteers. The journal of Alternative and Complementary Medicine. 2011; 17(9):823-826. 


\section{Bibliographic information of this paper for citing:}

Jometondoki H, Ghorat F, Rakhshani MH, Et al. The effect of topical application of lavender essential oil on pain se-verity during intravenous catheterization: a randomized clinical trial.

J Res Dev Nurs Midw, 2019; 16(2): 52-60.

Copyright @ 2019, Hamide Jometondoki, Fereshteh Ghorat, Mohammad Hassan Rakhshani, Hasan khalili. 